\title{
Can an integrated obstetric emergency simulation training improve respectful maternity care? Results from a pilot study in Ghana
}

\author{
Patience A. Afulani MBChB, MPH, PhD ${ }^{1}$ (D) | Raymond A. Aborigo MPH, PhD ${ }^{2}$ | \\ Dilys Walker MD ${ }^{1,3} \quad$ Cheryl A. Moyer MPH, PhD ${ }^{4} \quad$ Susanna Cohen MS, DNP, CNM, FAAN ${ }^{3,5}$ \\ John Williams MBChB, MA, MPH, PhD ${ }^{2}$
}

\footnotetext{
${ }^{1}$ School of Medicine, Institute for Global Health Sciences, University of California, San Francisco, California

${ }^{2}$ Navrongo Health Research Center, Navrongo, Ghana

${ }^{3}$ PRONTO International, Seattle, Washington

${ }^{4}$ University of Michigan, Ann Arbor, Michigan

${ }^{5}$ College of Nursing, University of Utah, Salt Lake City, Utah

\section{Correspondence}

Patience A. Afulani, School of Medicine, Institute for Global Health Sciences, University of California, San Francisco, CA. Email: Patience.Afulani@ucsf.edu

Funding information

This study was funded by United States Agency for International Development (USAID) and the USAID Systems for Health Project, cooperative agreement AID-641-A-14-00002.
}

\begin{abstract}
Background: Few evidence-based interventions exist on how to improve respectful maternity care (RMC) in low-resource settings. We sought to evaluate the effect of an integrated simulation-based training on provision of RMC.

Methods: The pilot project was in East Mamprusi District in northern Ghana. We integrated specific components of RMC, emphasizing dignity and respect, communication and autonomy, and supportive care, into a simulation training to improve identification and management of obstetric and neonatal emergencies. Forty-three providers were trained. For evaluation, we conducted surveys at baseline $(\mathrm{N}=215)$ and endline $(\mathrm{N}=318) 6$ months later, with recently delivered women to assess their experiences of care using the person-centered maternity care scale. Higher scores on the scale represent more respectful care.

Results: Compared to the baseline, women in the endline reported more respectful care. The average person-centered maternity care score increased from 50 at baseline to 72 at endline, a relative increase of $43 \%$. Scores on the subscales also increased between baseline and endline: $15 \%$ increase for dignity and respect, $87 \%$ increase for communication and autonomy, and 55\% increase for supportive care. These differences remained significant in multivariate analysis controlling for several potential confounders.
\end{abstract}

Conclusions: The findings suggest that integrated provider trainings that give providers the opportunity to learn, practice, and reflect on their provision of RMC in the context of stressful emergency obstetric simulations have the potential to improve women's childbirth experiences in low-resource settings. Incorporating such trainings into preservice and in-service training of providers will help advance global efforts to promote RMC.

\section{K E Y W O R D S}

Ghana, mistreatment, person-centered maternity care, quality of care, respectful maternity care, simulation training 


\section{1 | INTRODUCTION}

Respectful maternity care (RMC), which is core to personcentered care, is recognized as key to improving maternal and neonatal health outcomes. ${ }^{1,2}$ RMC is highlighted in the World Health Organization recommendations for a positive childbirth experience and is described as care during childbirth that maintains women's dignity; ensures privacy and confidentiality, and freedom from harm and mistreatment; and enables informed choice and continuous support during childbirth. ${ }^{3}$ Mistreatment or disrespect and abuse during childbirth represent lack of RMC, although the absence of disrespect and abuse is not equivalent to RMC. ${ }^{4,5}$ Growing evidence globally has highlighted non-RMC in health facilities, and its negative effects on health-seeking behavior and maternal and neonatal health outcomes. ${ }^{4-6}$ There is however limited evidence on how to improve it.

Studies in Africa suggest that multicomponent interventions can improve various aspects of RMC including reducing disrespect and abuse. ${ }^{7-11}$ These interventions include training health care providers in values and attitudes transformation and communication skills; setting up quality improvement teams; monitoring disrespect and abuse; improving staff conditions; maternity open days; dispute resolution, etc. ${ }^{12} \mathrm{~A}$ recent systematic review concluded that while these multicomponent interventions appear to reduce some aspects of disrespect and abuse, their sustainability is unclear and the intervention components with the greatest impact have not been identified. ${ }^{12}$ In addition, the heterogeneous nature of multicomponent interventions limits their feasibility and scalability in the context of limited resources. Thus, rigorous research to refine the optimum approach to deliver and achieve RMC in all settings is needed. ${ }^{12}$

Notably, these prior interventions were solely focused on improving RMC. ${ }^{7,8}$ Disrespectful care, however, does not exist in isolation; it often emerges in the process of providing highly stressful emergency care. Thus, interventions that address RMC in the context of providing stressful clinical care may be the most effective ways of improving it. Highly realistic clinical simulation training provides this unique opportunity to be responsive and respectful to women's needs in a meaningful context, while mimicking the stressful clinical environment that may contribute to disrespectful care. The potential effect of such a training is likely greater than the combined effect of stand-alone trainings on only clinical skills or only RMC. However, no studies to our knowledge have explicitly used this integrated simulation approach to improve RMC and documented the effect of the intervention on women's experiences. Thus, as part of a pilot study in Ghana to improve intrapartum quality of maternal and newborn care, we explicitly integrated concepts of RMC into a simulation-based provider training and evaluated the effect of the training on women's experiences. We present the evaluation results in this paper.

\section{2 | METHODS}

\section{1 | Study site and intervention}

The project was implemented in East Mamprusi District in the northern region of Ghana. The northern region has the highest maternal and infant mortality rates in Ghana. In 2016, the institutional maternal mortality ratio for northern Ghana was 208 per 100000 live births, compared to a national average of 164 per 100000 live births, ${ }^{13}$ and the infant mortality rate was 53 per 1000 live births compared to the national average of $41 .{ }^{14}$ The northern region also had the lowest rate of facility-based births at $35 \%$, compared to the national average of $73 \% .{ }^{14}$ Disrespectful care was a key factor driving low-facility delivery rates. ${ }^{6,15}$ East Mamprusi is a rural district with a population of about 121000 . The district has 13 health facilities, with approximately 114 providers, including four medical doctors, 88 nurses, 12 midwives, and 22 community health nurses. Seven of the facilities conduct deliveries, including one mission hospital serving as the district referral hospital, four health centers, and two smaller Community-based Health Planning and Services compounds. Collectively, these seven facilities oversee more than 5000 births per year (unpublished data, 2016). The pilot study was implemented at the five highest volume delivery facilities in the district, which were the referral hospital and four health centers.

We used provider trainings based on the methodology developed by PRONTO International: a low-tech, highly realistic simulation and team training with facilitated debriefing, to improve identification and management of obstetric and neonatal emergencies and team functioning. ${ }^{16-19}$ The PRONTO training kit, the PRONTOPack, includes a hybrid birth simulator called a PartoPants ${ }^{\mathrm{TM}}$ (a modified pair of surgical scrubs with anatomical landmarks necessary for delivery) worn by a patient actress (one of the female providers). The patient actress brings the patient to the center of the care and allows for direct discussion about patient experiences. Although PRONTO has always emphasized RMC before the current intervention, the PRONTO curriculum did not directly focus on RMC principles. In this project, we integrated RMC concepts into the curriculum and simulation scenarios in a deliberate way.

The curriculum for the training included five simulation scenarios and associated case-based learning modules and skills stations capturing seven priority topics identified during a stakeholder meeting (Table 1), plus interactive teamwork and communication activities. All simulations also emphasized various aspects of RMC, which highlighted treating women with dignity and respect, communicating with them, 
TA B L E 1 Training content for simulation-based provider training

Obstetrics and neonatal care topics

1. Normal birth practices and evidence-based maternity care

2. Immediate newborn evidence-based care

3. Neonatal resuscitation

4. Obstetric hemorrhage (postpartum hemorrhage and antepartum hemorrhage)

5. Preeclampsia/eclampsia

6. Sepsis

7. Preterm labor and birth

Aspects of RMC emphasized during training

1. Providers introducing themselves to the women

2. Calling women by their names

3. Asking women how they are feeling

4. Allowing women to have a support person of their choice

5. Ensuring privacy during examinations

6. Explaining examinations, procedures, and medications

7. Obtaining consent before procedures

8. Communicating findings of examinations to women and their families

9. Encouraging the women and their families to ask questions

10. Allowing women to move during labor and birth in their preferred position

respecting their autonomy, and supporting them in whatever way they needed including encouraging birth companions. The training content was based on evidence-based practices on the management of the complications and recommended practices known to have positive effects on birth outcomes and women's experiences such as support and mobility during labor and nonsupine position at delivery., ${ }^{3,20-25}$ In addition, simulation scripts had prompts for certain behaviors from the patient actress: For example, if providers did not introduce themselves, the patient actress asked "who are you?", and if providers did not explain what they were doing or found from examinations, she asked "what are you doing to me?" or "how is my baby?" Simulations were followed by a debrief to engage participants in guided self-analyses of their performance in the clinical management of the case as well as on their interactions with the patient and other medical personnel. During each debrief, the patient actress who was one of the participating providers was also asked to reflect on how she was treated during the simulation.

In addition, we included one simulation with a sole focus on RMC. This simulation involved a patient who initially refused to open her legs for examinations and then insisted on delivering in a squatting position. This simulation was followed by a debrief that emphasized RMC elements, such as how to communicate with patients who do not fit into perceived notions of cooperation ("difficult patients") to prevent verbal and physical abuse, and responding to women's desires for birthing in alternative positions. This simulation was paired with a clinical case review session, including a video to help providers understand the relevance of RMC and to demonstrate what RMC may look like in their setting. The providers also engaged in an interactive activity on RMC to help them understand and internalize the needs of women during childbirth.

Twenty-two providers from the intervention facilities first participated in a 2-day training facilitated by three PRONTO trainers at a location close to the referral hospital in April 2017. Six providers who participated in the first training were then invited to a 2-day simulation facilitator training (SFT) led by the PRONTO trainers. The goal of the SFT was to equip the participants with the knowledge and skills to become effective simulation facilitators to serve as trainers for the district. These new trainers then led an additional 2-day provider training, with support from the experienced PRONTO trainers, for an additional 21 providers, which included providers from the intervention district who had not participated in the first training and providers from the surrounding districts. This training involved the same content as the first provider training and enabled the local facilitators to gain confidence to facilitate simulations and moderate debriefing sessions. The local facilitators then conducted four 3-hour refresher trainings once a month at the intervention facilities between June 2017 and October 2017. Except for changes in timing of implementation and training location because of logistical issues, the protocol for the training was implemented as planned. Of the 43 providers trained, 22 were midwives, two were medical doctors, one was an anesthetist, and the remaining were nurses (including enrolled, public, and community health nurses). Most providers (72\%) of the 35 providers who filled out a baseline survey had never participated in a simulation-based training before this training.

\section{2 | Data collection, measures, and analysis}

To evaluate the effect of the training on RMC, we conducted interviews with recently delivered women in the five intervention facilities before and after the intervention. Our planned sample size was 300 women at each time point, which we estimated would detect an effect size of 0.45 (assuming $80 \%$ power and five clusters (health facilities)), and the assumption that we could recruit about half of eligible women in the intervention facilities. Women were eligible if they were aged 15-49 years and delivered in a health facility within the preceding 8 weeks. Research assistants approached women as they exited the health facilities after they had received care and invited them to participate in the study. All eligible women contacted consented to participate. The interviews were then conducted by the research assistants in the local languages (Mampruli and Kokomba) at the facility or the woman's home. The baseline survey was conducted in March and April 2017 just before the initial provider training and the endline conducted in November 2017, 6 months after the initial provider training and 1 month after the fourth refresher. A total of 268 and 320 women were interviewed 
at baseline and endline, respectively. We restricted the analytic sample to women who had complete information on the outcome variable $(\mathrm{N}=215$ for baseline and 318 endline). All participants provided written informed consent. The study was approved by the ethics review boards of the University of California, San Francisco, and the Navrongo Health Research Center in Ghana and deemed exempt at the University of Michigan.

Our measure of RMC (the dependent variable) was the score on the person-centered maternity care scale. The person-centered maternity care scale was initially validated in Kenya and India and shown to have high content, construct, and criterion validity and with good reliability. ${ }^{26,27}$ The original scale has 30 items with three subscales for dignity and respect, communication and autonomy, and supportive care. Each item has a 4-point frequency response option-0: "no, never," 1: "yes, a few times," 2: "yes, most of the time," and 3: "yes, all the time." Minor modifications were made to the wording of one question during pretesting in Ghana. Exploratory factor analysis using both the baseline and endline data supported a three-factor structure with a single dominant factor. Three items (time to care, delivery support, and crowding), however, had low loadings $(<0.1)$ in the one factor structure analysis. Thus, we decided to exclude these three items from the scale. We also excluded three items on availability of water, electricity, and perception of enough staff since the intervention did not include improvements to infrastructure or number of providers. The analysis was therefore based on a 24-item version of the scale. The items excluded were all part of the supportive care subscale, decreasing the number of items in that subscale from 15 in the original scale to 9 in the 24 -item version. The dignity and respect and communication and autonomy subscales have six and nine items, respectively, as in the original scale. The full 24-item scale and subscales have good internal consistency, with Cronbach's $\alpha$ of 0.9 for the full scale and over 0.7 for the subscales. We summed items in the full scale and subscales (with negative items reverse coded) to generate person-centered maternity care and subscale scores. To enable comparison across the domains, we rescaled the scores-scores shown as a fraction of the total possible score on that domain multiplied by 100 , which puts each score between 0 (lowest quality) and 100 (best quality).

The key independent variable was the time of data collection in relation to the intervention, with options as baseline (before the intervention) or endline (after the intervention). We also collected data on confounders including demographic, health, and socioeconomic factors as well as facility and provider characteristics, which have been shown in previous studies to be associated with women's experiences, ${ }^{28,29}$ and could differ for the baseline and endline samples.

For the analysis, we first examined the distribution of variables for the baseline and endline samples using descriptive and bivariate analysis. Next, we examined the distributions of the individual items in the scale using chi-squared test to assess differences between the baseline and endline responses. We then generated the full-scale and subscale scores and examined mean differences in scores between the baseline and endline using two-sample $t$ tests. Because our outcome variables (scores on the scale and subscales) were continuous, we used ordinary least-squares regressions (bivariate and multivariate linear regressions) to examine the differences in scores at baseline and endline. We controlled for confounders in the multivariate models by including all variables that were associated with scores in the bivariate models or which had strong theoretical rationale for inclusion. The beta coefficients obtained from the linear regression models represent the degree of change in the scale scores for every one unit of change in continuous predictors, or the difference between any category and the reference category for categorical variables. ${ }^{30}$ The coefficients for the endline in the multivariate models therefore represent differences between the scores at endline and baseline, controlling for potential confounders. The positive scores represent increases in the scores. We also ran sensitivity analysis using multivariate multilevel linear regression models with random intercepts at the individual and facility level, to assess whether using that method of accounting for clustering within facilities changed the results. ${ }^{31,32}$ We used STATA 15 (StataCorp LLC, College Station, TX, USA) for the analysis.

\section{\begin{tabular}{l|l}
3 & RESULTS
\end{tabular}}

Small but statistically significant differences were found in the characteristics of women interviewed in the baseline and endline (Table 2). For example, women in the endline were more likely to be younger and primiparous (average age was 27 years with $31 \%$ primiparous in the endline compared to average age of 30 years and $19 \%$ primiparous in the baseline). Compared to women in the baseline, women in the endline also were slightly more educated and literate, from wealthier households, and their partners had more education.

With a few exceptions, the responses on most of the individual items suggest women in the endline received more respectful care than those at baseline (Table 3; Table S1). For example, only $12 \%$ of women at baseline felt they were treated with respect all the time, and $8 \%$ felt they were treated in a friendly manner all the time compared to $64 \%$ and $65 \%$, respectively, at endline. At baseline, $87 \%$ of women reported providers never introduced themselves to them, and $43 \%$ reported providers never called them by their names, compared to $60 \%$ at and $20 \%$, respectively, at endline. Over 50\% also reported providers did not explain the purpose of examinations, procedures, or medications at baseline compared to $<25 \%$ at endline, and $43 \%$ reported providers never asked for permission before examinations and procedures at baseline, compared to 
TABLE 2 Characteristics of women, East Mamprusi District, Ghana, 2017

\begin{tabular}{|c|c|c|c|}
\hline & $\begin{array}{l}\text { Baseline } \\
(\mathrm{N}=\mathbf{2 1 5})\end{array}$ & $\begin{array}{l}\text { Endline } \\
(\mathrm{N}=\mathbf{3 1 8})\end{array}$ & \\
\hline & $\mathbf{N}(\%)$ & $\mathbf{N}(\%)$ & $P$-value \\
\hline \multicolumn{4}{|l|}{ Intervention facility } \\
\hline Referral hospital & $37(17.2)$ & $100(31.4)$ & \multirow[t]{5}{*}{0.00} \\
\hline Health center 1 & $51(23.7)$ & $54(17.0)$ & \\
\hline Health center 2 & $39(18.1)$ & $66(20.8)$ & \\
\hline Health center 3 & $56(26.0)$ & $60(18.9)$ & \\
\hline Health center 4 & $32(14.9)$ & $38(11.9)$ & \\
\hline \multicolumn{4}{|l|}{ Age } \\
\hline 15 to 19 years & $11(5.1)$ & $48(15.1)$ & \multirow[t]{4}{*}{0.00} \\
\hline 20 to 29 years & $102(47.7)$ & $156(49.1)$ & \\
\hline 30 to 48 years & $101(47.2)$ & $114(35.8)$ & \\
\hline Currently married & $202(94.0)$ & $288(90.6)$ & \\
\hline \multicolumn{4}{|l|}{ Parity } \\
\hline 1 & $40(18.9)$ & $97(30.8)$ & \multirow[t]{4}{*}{0.04} \\
\hline 2 & $48(22.6)$ & $60(19.0)$ & \\
\hline 3 & $38(17.9)$ & $54(17.1)$ & \\
\hline 4 to 9 & $86(40.6)$ & $104(33.0)$ & \\
\hline \multicolumn{4}{|l|}{ Highest education } \\
\hline No school/primary & $157(73.0)$ & $205(64.5)$ & \multirow[t]{3}{*}{0.05} \\
\hline Post-primary/vocational/secondary & $55(25.6)$ & $100(31.4)$ & \\
\hline College or above & $3(1.4)$ & $13(4.1)$ & \\
\hline Literate (able to read and write) & $7(3.3)$ & $49(15.4)$ & 0.00 \\
\hline \multicolumn{4}{|l|}{ Household wealth quintile } \\
\hline Poorest & $62(30.2)$ & $73(23.1)$ & \multirow[t]{4}{*}{0.00} \\
\hline Poorer & $57(27.8)$ & $106(33.5)$ & \\
\hline Middle & $80(39.0)$ & $103(32.6)$ & \\
\hline Richer/richest & $6(2.9)$ & $34(10.7)$ & \\
\hline \multicolumn{4}{|l|}{ Partner's education } \\
\hline No school/primary & $156(73.2)$ & $184(58.8)$ & \multirow[t]{4}{*}{0.01} \\
\hline Post-primary/vocational/secondary & $34(16.0)$ & $79(25.2)$ & \\
\hline College or above & $18(8.5)$ & $39(12.5)$ & \\
\hline No partner & $5(2.3)$ & $11(3.5)$ & \\
\hline \multicolumn{4}{|l|}{ Delivery provider type } \\
\hline Nurse/midwife & $184(85.6)$ & $245(77.0)$ & \multirow[t]{4}{*}{0.00} \\
\hline Doctor/medical officer & $18(8.4)$ & $29(9.1)$ & \\
\hline Nonskilled attendant & $10(4.7)$ & $1(0.3)$ & \\
\hline 1 or more skilled providers & $3(1.4)$ & $43(13.5)$ & \\
\hline \multicolumn{4}{|l|}{ Delivery provider sex } \\
\hline Male & $11(5.1)$ & $31(9.7)$ & 0.15 \\
\hline Female & $201(93.9)$ & $285(89.6)$ & \\
\hline Both & $2(0.9)$ & $2(0.6)$ & \\
\hline Has health insurance & $209(97.2)$ & $312(98.1)$ & 0.49 \\
\hline Had any complications & $107(49.8)$ & $188(59.1)$ & 0.03 \\
\hline Prior facility delivery & $156(72.6)$ & $205(64.5)$ & 0.05 \\
\hline
\end{tabular}




\begin{tabular}{|lccc|} 
& $\begin{array}{l}\text { Baseline } \\
(\mathbf{N = 2 1 5})\end{array}$ & $\begin{array}{l}\text { Endline } \\
(\mathbf{N}=\mathbf{3 1 8})\end{array}$ & \\
& $\mathbf{N}(\%)$ & $\mathbf{N}(\%)$ & $\boldsymbol{P}$-value \\
\hline $\begin{array}{l}\text { Self or household member work in health } \\
\text { facility }\end{array}$ & $18(8.4)$ & $56(17.6)$ & 0.00 \\
\hline First antenatal visit in first trimester & $164(76.6)$ & $220(69.2)$ & 0.24 \\
\hline $\begin{array}{l}\text { or more antenatal visits } \\
\text { Mampruli ethnicity }\end{array}$ & $196(92.0)$ & $287(90.8)$ & 0.36 \\
\hline Religious affiliation & $102(47.4)$ & $175(55.0)$ & 0.09 \\
\hline Muslim & $161(75.9)$ & $261(82.6)$ & 0.01 \\
\hline Christian & $51(24.1)$ & $46(14.6)$ & \\
\hline Other & $0(0.0)$ & $9(2.8)$ & \\
\hline Postpartum length $<5$ weeks & $154(71.6)$ & $177(55.7)$ & 0.00 \\
\hline
\end{tabular}

TABLE 2 (Continued)

$P$-value indicates significance of difference between baseline and endline. Household wealth is calculated from a wealth index based on 13 questions on household assets from the equity tool developed by metrics for measurement. ${ }^{36}$ The distribution across the wealth quintiles is not equal because the calculation is weighted to reflect the wealth quintile a participant will fall into when compared to other people in the country, not the sample.

$11 \%$ at endline. Over half (59\%) did not feel they could adopt a birthing position of their choice during delivery at baseline compared to $31 \%$ at endline. Women were more likely to be allowed to have labor companions at endline than at baseline: $32 \%$ reported they were never allowed to have a companion during labor at baseline compared to $10 \%$ at endline.

The full-scale and subscale scores also suggest women in the endline received more respectful care than those at baseline (Table S2 and Figure 1). The average rescaled personcentered maternity care score increased from 50 at baseline to 72 at endline, a relative increase of $43 \%$. Scores on the subscales also increased between baseline and endline: from 76 to 87 for dignity and respect (15\% relative increase), 31 to 58 for communication and autonomy ( $87 \%$ relative increase), and 52 to 75 for supportive care (45\% relative increase). Person-centered maternity care scores increased between baseline and endline in all facilities.

The differences between the baseline and endline scores remained significant in the multivariate analysis (Table 4). After controlling for several potential confounders, the endline person-centered maternity care score was about 18 points higher than the baseline scores $(\beta=17.6$; 95\% $\mathrm{CI}=15.6-19.6)$. Controlling for other factors, the differences between the baseline and endline scores for dignity and respect, communication and autonomy, and supportive care were $2.4,7.7$, and 7.4 , respectively. The results were essentially the same in the multilevel analysis. The multivariate analysis also showed that in general women received more respectful care in the health centers than in the referral hospital (Table S3). In addition, women's experiences differed by various factors including parity, literacy, household wealth, employment, and partner's education and employment.

\section{DISCUSSION}

After implementation of an integrated, low-tech, high-fidelity obstetric emergency simulation training in northern Ghana, RMC measured with the person-centered maternity care scale was substantially higher at endline than at baseline. The findings suggest that integrated low-tech high-fidelity simulation trainings have the potential to improve RMC in low-resource settings. It adds to the growing research suggesting that interventions targeting RMC can improve women's childbirth experiences. Specifically, it highlights that situating RMC in the context of broader quality of care initiatives may have great potential to improve women's childbirth experiences.

The highest change was in the domain of communication and autonomy, where the score almost doubled. A potential reason for this is that the PRONTO training has an emphasis on teamwork and communication and all simulations and debriefings included various elements of provider-provider and provider-patient communication. The training also emphasized patient autonomy, including asking for consent and respecting patient preference for delivering in alternative positions. One provider even shared a picture after the training showing how she had been able to assist a woman deliver in her preferred position, which was on a sheet on the floor instead of the delivery bed.

Supportive care also increased substantially, as this was emphasized in the form of asking women how they were feeling and having birth companions in all simulations. In debriefings, however, discussion of constraints of having a companion in the delivery room (eg, privacy when two women are delivering at the same time) led to compromises 
T A B L E 3 Percent of women responding "Yes, most of the time" or "Yes, all the time" to items in the person-centered maternity care scale, East Mamprusi District, Ghana, 2017

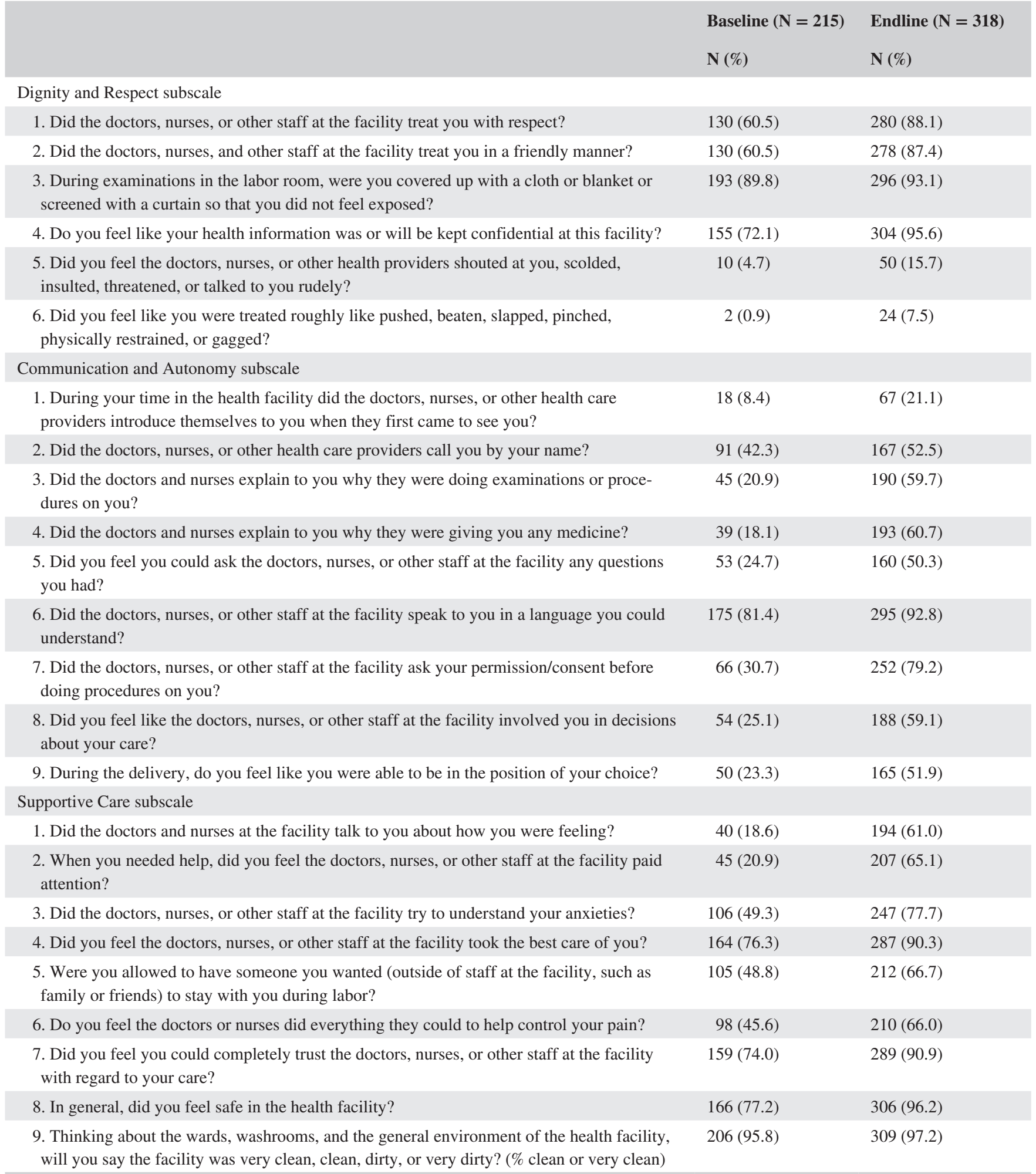

All differences between baseline and endline scores are significant at $P<0.001$ or $<0.01$.

of at least allowing companions during labor, where they could provide support not only to the woman, but also to the provider. These challenges of providing continuous support are described in detail elsewhere. ${ }^{33}$
The smallest change was in dignity and respect. Potential reasons for this include the relatively high scores for dignity and respect at baseline. In addition, reports of verbal and physical abuse paradoxically increased, despite the increase in reports 


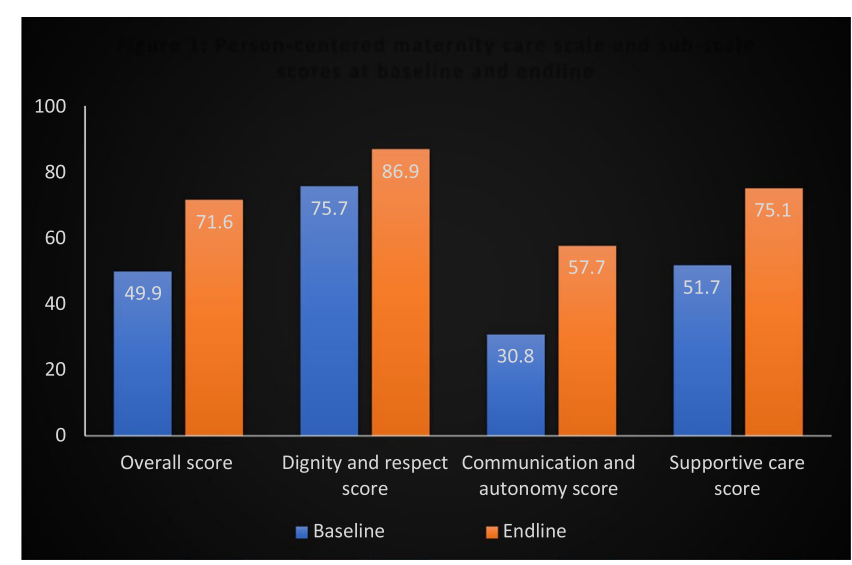

F I G URE 1 Person-centered maternity care scale and subscale scores at baseline and endline. Notes: These are the rescaled scores, so the range for each is from 0 to 100 . The differences are statistically significant $(P<0.001)$ [Colour figure can be viewed at wileyonlinelibrary.com]

of being treated with respect. Such contradictory effects have been observed in some prior studies when examining individual aspects of disrespect and abuse. ${ }^{12}$ One potential reason is that, while treating women with dignity and respect was emphasized in the training, verbal and physical abuse never actually occurred in the simulations. Thus, there was no opportunity for discussion of abuse in the debriefings - except for after the simulation with a "difficult patient" in which facilitators brought up the issue of abuse in the context of how providers might respond when they deem a patient as difficult. Prevention of abuse was therefore not reinforced in the training, which was a weakness of the training. The socioeconomic differences between women in the baseline and endline may also have contributed to this finding, as women of higher socioeconomic status may be more likely to report mistreatment than women of lower socioeconomic status. ${ }^{34}$ The effect of the training may therefore be potentially higher than estimated from these surveys.

The observed effects should be considered in light of the fact that this study did not include any effort to change existing infrastructure (such as lack of screens for privacy) or to address systemic issues (such as provider shortage and lack of supplies) that might make practicing in this setting difficult for providers. Such issues, while important to maintaining a motivated workforce that can in turn provide high-quality respectful care, are beyond the scope of training-based interventions. But they are crucial to creating sustainable change.

This study also adds to the growing evidence on predictors of RMC. The higher person-centered maternity care scores for women of higher socioeconomic status (literate, employed, and wealthier) and those who delivered in lowerlevel facilities (compared to women of lower socioeconomic status and those who delivered in the higher-level facilities) are consistent with findings from studies in Kenya and India. ${ }^{28,29,34}$ The potential reasons for these disparities have been described in detail elsewhere ${ }^{28,34,35}$ and include literate women being more empowered to advocate for themselves, employed and wealthier women having the resources to access facilities that provide higher quality care, and differential positive treatment of women of higher socioeconomic status. Higher patient loads and lower social costs to providers in higher-level facilities who mistreat women (because they may be less easily identified and have limited interactions with the communities they serve) may also account for the less respectful care in these facilities. For the purpose of evaluation, these disparities highlight the need to collect data on and account for factors that might affect women's experiences and their reporting.

There have been a limited number of studies assessing the effects of an intervention on RMC in sub-Saharan Africa, and to our knowledge, ours is the first to do so in the context of a clinical simulation training. There are however several limitations to this study. First, funding limitations precluded our recruitment of a control group; thus, it is possible that other external factors could account for the results given the increasing interest in RMC globally. There were, however, no other specific activities targeting RMC in the intervention district during the project period; thus, we believe the training

TA B L E 4 Multivariate regression of person-centered maternity care scale and subscale scores, East Mamprusi District, Ghana, 2017 $(\mathrm{N}=499)$

\begin{tabular}{|c|c|c|c|c|}
\hline & Overall score & Dignity and respect score & $\begin{array}{l}\text { Communication and } \\
\text { autonomy score }\end{array}$ & $\begin{array}{l}\text { Supportive care } \\
\text { score }\end{array}$ \\
\hline & Beta coefficient (95\% CI) & Beta coefficient (95\% CI) & Beta coefficient (95\% CI) & $\begin{array}{l}\text { Beta coefficient } \\
(95 \% \text { CI })\end{array}$ \\
\hline \multicolumn{5}{|c|}{ Data collection period } \\
\hline Baseline & Reference & Reference & Reference & Reference \\
\hline Constant & $24.2 * * *(13.6-34.8)$ & $9.8 * * *(6.7-13.0)$ & $6.9 *(1.6-12.3)$ & $7.4 * *(2.9-12.0)$ \\
\hline
\end{tabular}

Model controls for facility, age, parity, marital status, literacy, household wealth, occupation, partner's education and occupation, insurance status, complications, prior facility delivery, timing and frequency of antenatal care, position and sex of delivery provider, religion, tribe, and timing of interviews.

$* P<0.05 ; * * P<0.01 ; * * * P<0.001$. 
accounts for most of the effects. Second, not all providers in the intervention facilities were exposed to both the initial trainings and refreshers because of workforce turnover. The observed effect could therefore be smaller than the potential effect of the intervention. Third, given the short timeline for the intervention and evaluation (6 months), we are unable to assess long-term sustainability. Fourth, interviewers were not blinded to the study and this could have affected how interviewers asked questions or interpreted women's responses.

In addition, the evaluation data presented are based on cross-sectional surveys with different groups of women, meaning that other factors that affect reporting of women's experiences could explain some of the results. However, given that the findings are significant after controlling for other potential predictors, it is not likely that these other factors can explain all of the observed associations. It was also not possible to conduct longitudinal data collection from the same group of women as the same women were unlikely to receive maternity care within the project period. Because the data are based on self-reporting, social desirability and recall bias are potential issues. Women's reports of their experiences are also often influenced by their expectations, which could result in women reporting respectful care, even when they have been mistreated. However, self-reports are a valid source for assessing people's experiences as their interpretation of the event may be more likely to affect their response to the encounter than what actually happened. In addition, the use of a validated multidimensional scale helps to reduce bias based on responses to individual items. Finally, the findings may not be generalizable to other settings given unique aspects of the study district. Nonetheless, we believe this intervention could be adapted to many lowresource settings.

\section{1 $\mid$ Conclusions}

These findings highlight the feasibility and potential effectiveness of integrated low-tech, high-fidelity simulation trainings to improving RMC. The findings suggest that trainings that give providers the opportunity to learn, practice, and reflect on their provision of RMC in the context of providing stressful emergency care have the potential to improve women's experiences in developing settings. Incorporating such trainings into preservice and in-service training of providers may advance global efforts to promote RMC. Future research is needed to more rigorously evaluate the effect of the intervention on not just RMC, but also on other maternal and neonatal health outcomes such as health-seeking behaviors, morbidity, and mortality. Studies based on more rigorous methodologies such as cluster randomized controlled trials, as well as longer and larger-scale studies, are needed to assess effectiveness, sustainability, and scaling mechanisms. Cost-effectiveness studies are also needed. Such research would provide stronger evidence to advocate for government uptake for scalability and sustainability.

\section{ACKNOWLEDGMENTS}

This study was made possible by the support of the American people through the United States Agency for International Development (USAID). The findings of this study are the sole responsibility of the authors and do not necessarily reflect the views of USAID or the United States Government.

We will like to thank all members of our project team, our trainers, the providers who participated in the training, the field staff who conducted the surveys, and all the women who agreed to be interviewed.

\section{ORCID}

Patience A. Afulani (D) https://orcid.org/0000-0002-6739-234X

\section{REFERENCES}

1. Miller S, Abalos E, Chamillard M, et al. Beyond too little, too late and too much, too soon: a pathway towards evidence-based, respectful maternity care worldwide. Lancet. 2016;388:2176-2192.

2. Tunçalp Ö, Were W, MacLennan C, et al. Quality of care for pregnant women and newborns-the WHO vision. BJOG Int J Obstet Gynaecol. 2015;122(8):1045-1049.

3. World Health Organization. WHO recommendations: intrapartum care for a positive childbirth experience. WHO. 2018. http://www. who.int/reproductivehealth/publications/intrapartum-care-guidelines/en/. Accessed February 28, 2018.

4. Bohren MA, Vogel JP, Hunter EC, et al. The mistreatment of women during childbirth in health facilities globally: a mixedmethods systematic review. PLoS Medicine. 2015;12(6):e1001847.

5. Shakibazadeh E, Namadian M, Bohren MA, et al. Respectful care during childbirth in health facilities globally: a qualitative evidence synthesis. BJOG Int J Obstet Gynaecol. 2017;125:932-942.

6. Bohren MA, Hunter EC, Munthe-Kaas HM, Souza JP, Vogel JP, Gülmezoglu AM. Facilitators and barriers to facility-based delivery in low- and middle-income countries: a qualitative evidence synthesis. Reprod Health. 2014;11(1):71.

7. Ratcliffe HL, Sando D, Lyatuu GW, et al. Mitigating disrespect and abuse during childbirth in Tanzania: an exploratory study of the effects of two facility-based interventions in a large public hospital. Reprod Health. 2016;13:79.

8. Abuya T, Ndwiga C, Ritter J, et al. The effect of a multi-component intervention on disrespect and abuse during childbirth in Kenya. BMC Pregnancy Childbirth. 2015;15(1):224.

9. Kujawski SA, Freedman LP, Ramsey K, et al. Community and health system intervention to reduce disrespect and abuse during childbirth in Tanga Region, Tanzania: a comparative before-andafter study. PLoS Medicine. 2017;14(7):e1002341.

10. Umbeli T, Murwan IO, Kunna A, Ismail S, Sulman MM, Elmahgoub A. Impact of health care provider's training on patients' communication during labor at Omdurman Maternity Hospital, Sudan 2011. Sudan J Med Sci. 2014;9(4):211-216. 
11. Brown H, Hofmeyr GJ, Nikodem VC, Smith H, Garner P. Promoting childbirth companions in South Africa: a randomised pilot study. BMC Med. 2007;5(1):7.

12. Downe S, Lawrie TA, Finlayson K, Oladapo OT. Effectiveness of respectful care policies for women using routine intrapartum services: a systematic review. Reprod Health. 2018;15:23.

13. Ghana Health Service. Ghana Health Service 2016 annual report. 2017. http://www.ghanahealthservice.org/publications.php.

14. Ghana Statistical Service, Ghana Health Service. Ghana Demographic and Health Survey 2014: key indicators. Accra: Ghana Statistical Service, Ghana Health Service, 2015.

15. Moyer CA, Adongo PB, Aborigo RA, Hodgson A, Engmann CM. "They treat you like you are not a human being": maltreatment during labour and delivery in rural northern Ghana. Midwifery. 2014;30(2):262-268.

16. Cohen SR, Cragin L, Rizk M, Hanberg A, Walker DM. PartoPants $^{\mathrm{TM}}$ : The high-fidelity, low-tech birth simulator. Clin Simul Nurs. 2011;7(1):e11-e18.

17. Cohen SR, Cragin L, Wong B, Walker DM. Self-efficacy change with low-tech, high-fidelity obstetric simulation training for midwives and nurses in Mexico. Clin Simul Nurs. 2012;8(1):e15-e24.

18. Fahey JO, Cohen SR, Holme F, et al. Promoting cultural humility during labor and birth: putting theory into action during PRONTO obstetric and neonatal emergency training. J Perinat Neonatal Nurs. 2013;27(1):36-42.

19. Walker D, Cohen S, Fritz J, et al. Team training in obstetric and neonatal emergencies using highly realistic simulation in Mexico: impact on process indicators. BMC Pregnancy Childbirth. 2014; $14: 367$.

20. Afulani PA, Kirumbi L, Lyndon A. What makes or mars the facility-based childbirth experience: thematic analysis of women's childbirth experiences in western Kenya. Reprod Health. 2017; 14:180.

21. Downe S, Finlayson K, Oladapo O, Bonet M, Gülmezoglu AM. What matters to women during childbirth: a systematic qualitative review. PLoS ONE. 2018;13(4):e0194906.

22. Bohren MA, Hofmeyr GJ, Sakala C, Fukuzawa RK, Cuthbert A. Continuous support for women during childbirth. Cochrane Database Syst Rev. 2017;7:CD003766.

23. Lawrence A, Lewis L, Hofmeyr GJ, Styles C. Maternal positions and mobility during first stage labour. Cochrane Database Syst Rev. 2013;(8):CD003934.

24. DiFranco JT, Curl M. Healthy birth practice \#5: avoid giving birth on your back and follow your body's urge to push. J Perinat Educ. 2014;23(4):207-210.

25. WHO recommendation on birth position (for women without epidural analgesia) I RHL. https://extranet.who.int/rhl/topics/ preconception-pregnancy-childbirth-and-postpartum-care/careduring-childbirth/care-during-labour-2nd-stage/who-recommendation-birth-position-women-without-epidural-analgesia. Accessed November 30, 2018.
26. Afulani PA, Diamond-Smith N, Golub G, Sudhinaraset M. Development of a tool to measure person-centered maternity care in developing settings: validation in a rural and urban Kenyan population. Reprod Health. 2017;14(1):118.

27. Afulani PA, Diamond-Smith N, Phillips B, Singhal S, Sudhinaraset M. Validation of the person-centered maternity care scale in India. Reprod Health. 2018;15(1):147.

28. Afulani PA, Sayi TS, Montagu D. Predictors of person-centered maternity care: the role of socioeconomic status, empowerment, and facility type. BMC Health Serv Res. 2018;18(1):360.

29. Afulani PA, Phillips B, Aborigo RA, Moyer CA. Person-centred maternity care in low-income and middle-income countries: analysis of data from Kenya, Ghana, and India. Lancet Glob Health. 2019;7(1):e96-e109.

30. Miller JE. The Chicago Guide to Writing about Multivariate Analysis. Chicago, IL: University of Chicago Press; 2005.

31. Hox JJ. Multilevel Analysis. London: Routledge Academic; 2010.

32. Rabe-Hesketh S, Skrondal A. Multilevel and Longitudinal Modeling Using Stata, Volumes I and II, 3rd edn. College Station, TX: Stata Press; 2012.

33. Afulani P, Kusi C, Kirumbi L, Walker D. Companionship during facility-based childbirth: results from a mixed-methods study with recently delivered women and providers in Kenya. BMC Pregnancy Childbirth. 2018;18:150.

34. Sudhinaraset M, Treleaven E, Melo J, Singh K, Diamond-Smith N. Women's status and experiences of mistreatment during childbirth in Uttar Pradesh: a mixed methods study using cultural health capital theory. BMC Pregnancy Childbirth. 2016;16:332.

35. Afulani PA. Rural/Urban and socioeconomic differentials in quality of antenatal care in Ghana. PLoS ONE. 2015;10(2):e0117996.

36. Metrics for measurement. EquityTool. M4MGMT. 2016. http:// m4mgmt.org/allprojects/the-equity-tool. Accessed June 14, 2017.

\section{SUPPORTING INFORMATION}

Additional supporting information may be found online in the Supporting Information section at the end of the article.

How to cite this article: Afulani PA, Aborigo RA, Walker D, Moyer CA, Cohen S, Williams J. Can an integrated obstetric emergency simulation training improve respectful maternity care? Results from a pilot study in Ghana. Birth. 2019;46:523-532. https://doi. org/10.1111/birt.12418 